\title{
BIBLIOGRAFIA
}

\section{Hacia una Bibliografía de y sobre Carlos Fuentes*}

I. OBRAS DE CARLOS FUENTES 1

A. Cuentos

r. Los días enmascarados (México: Studium, 1954).

2. Cantar de ciegos (México: Joaquín Mortiz, 1964).

B. NOVELAS ${ }^{2}$

I. La región más transparente (México: Fondo de Cultura Económica, I958).

Segunda edición (Idem., 1958),

Tercera edición (Idem., I960),

Cuarta edición (Idem., I963).

* Beca de Canada Council, verano de 1964.

1 Fondo de Cultura Económica proyecta publicar otra novela suya, El béroe sin vida, pero hasta hoy no ha llegado a aparecer. También está preparando actualmente un libro de ensayos.

2 Se han publicado sus obras en otros países y otras lenguas. Por ejemplo, The Death of Artemio Cruz apareció recientemente traducida al inglés (New York: Farrat, Straus \& Co.). Es la tercera novela suya publicada en los Estados Unidos. Las otras en edición inglesa son Where the Air is Clear (New York: Ivan Obolensky, Inc., 1960) y The Good Conscience (New York: Ivan Obolensky Inc., 1961). 
2. Las buenas conciencicis ${ }^{3}$ (México: Fondo de Cultura Económica, 1959). Segunda edición (Idem., r960), Tercera edición (Idem., r961).

3. La muerte de Artemio Cruz (México: Fondo de Cultura Económica, I962).

4. Aura (México Era, I962).

C. Prólogos, Artícullos, Reseñas y ENsayos

I. "México y los Estados Unidos", Universidad de México, No 7 (1959).

2. "Party of One", Holiday, XXXII (I962).

3. "Punat del Este", Nation, CXCIV (1962).

4. "Prólogo", en El cine y el bombre contemporáneo de M. Michel (México: Universidad Veracruzana, 1962).

5. "The Argument of Latin America: Words for the North Americans," Whither Latin America (New York: Monthly Review Press, 1963), pp. 9-24.

6. "A Life", The New York Review, June 25, 1964, Pp. 2-4 (reseña sobre $A$ Mexican Peasant and His Family de Pedro Martínez).

7. "La nueva novela latinoamericana", Siempre, 579, 29 de julio de 1964.

\section{SOBRE CARLOS FUENTES}

I. Adams, Mildred, "The Line of Life Lies Between Paralysis and Frenzy", New York Times Book Review, May 24, I964, p. 4 (sobre The Death of Artemio Cruz).

2. Agüero, Luis, "Carlos Fuentes, 'Aura" ", Casa de las Américas, Año II, Nos. I5-I6 (noviembre de I962-febrero de I963), PP. 40-42.

3. Alegría, Fernando, "Carlos Fuentes", Novelistas contemporáneos bis. panoamericanos (Boston: D. C. Heath, I964), Pp. II5-II6.

4. Alvarado, José, "Correo menor", Excelsior: Diorama de la Cultura, 22 de junio de 1958 .

3 Las buenas conciencias es la primera parte de una tetralogía, cuatro novelas reunidas con el título "Los Nuevos"; las siguientes aun no publicadas: La patria de nadie, Guadalupe Villegas, y Los grandes intereses.

4 Esta lista sólo hace accesible una selección del creciente número de sus artículos, ensayos, etc., que van apareciendo en México y en los Estados Unidos. Carlos Fuentes, en la actualidad, colabora en muchas revistas en los dos países. Para el lector que desee enterarse de sus artículos socioeconómicos, véase particularmente Universidad de México, Siempre, The Nation. Asimismo escenógrafo para la industria cinematográfica, acaba de terminar con Luis Buñuel los diảlogos de la película El acacaso, según la novela de Alejo Carpentier de igual título. 
5. Alvarez, A., "Mortal Longings", New York Review of Books, II (June II, I964), r4-16 (sobre The Deatb of Artemio Cruz).

6. Andrés Murillo, Luis, "Carlos Fuentes, La región más transparente", Revista Iberoamericana (1960), pp. I94-196.

7. Campos, Jorge, "La sociedad mejicana en un nuevo novelista: Carlos Fuentes", Insula, $\mathrm{N}^{\circ}$ I96, p. I3 (sobre La región mâs transparente y Las buenas conciencias).

8. Carballo, Emmanuel, "Conversación con Carlos Fuentes", Novedades: México en la Cultura, is de noviembre de 1959 , p. 12.

9. Castellanos, Rosario, "La novela mejicana contemporánea y su valor testimonial", Hispania, XLVII, N"2 (May I964), 223-230.

rc. Chavarri, Raúl, "La novela moderna mejicana", Cuadernos Hispanoamericanos, N* I73 (mayo de I964), pp. 367-379.

rr. Couffon, Claude, "Carlos Fuentes y la novela mexicana", Cuadernos Congreso por la Libertad de la Cultura, No 42 (mayo-junio de I960), pp. 67-69.

12. Curley, Thomas, "Professed Christians in the Role of Pharisees", The Commonwed, LXXV (Jan. I9, I962), 439-440 (sobre The Good Conscience).

13. Díaz-Lastra, Adalberto, "Carlos Fuentes: La región más transparente", Cuadernos Hispanoamericanos, Nos. $175-176$ (julio-agosto de I964), pp. 242-247.

14. Durán, Manuel, "Cuatro novelas mexicanas", Revista Hispánica Moderna, XXVI (1960), I43-r45 (sobre Yáñez, Benítez, Fuentes, Galindo).

15. González Arauzo, A., "No Other Ends Than Possession", Mexico Quarterly Review (1962), pp. 268-27I (sobre La muerte de Artemio Cruz).

r6. Kielly, Gerald, "Kaleidoscopic Effects", Mexico Quarterly Review, (I960), pp. 2ro-2I2.

17. Lane, Helen, "Last Rites for a Fat Cat", Nation, CXCVIII (June r964), 558 (sobre The Death of Artemio Cruz).

18. Leal, Luis, "Contemporary Mexican Literature: A Mirror of Social Change", University of Arizona Quarterly, XVIII, N:3 (Autumn I962), I97-207.

I9. Leiva, Raúl, "Tres grandes novelas mexicanas en I962", Cuadernos de Bellas Artes, IV, No I (1963), 25-34 (sobre Fuentes, La muerte de Artemio Cruz; Castellanos, Oficio de Tinieblas; Yáñez, Las tierras flacas). 
20. Maldonado Denis, M., "Sobre Carlos Fuentes", Cuadernos Hispanoamericanos, III, No 9 ( 1963 ), 63-66.

2x. Maloff, Saúl, "Growing Pains of a Bourgeois", Salurday Review. XIIV (Dec. I6, I96r), 20-2I (sobre The Good Conscience).

22. "The Marxist Myth of Mexico", reseña anónima, Time, June 5, 1964, p. 68 (sobre The Death of Artemio Cruz).

23. Mead, Jr., Robert G., "Aspects of Mexican Literature Today", Books Abrowd, XXXIV, No I (Winter 1960), 5-8.

24. - "Carlos Fuentes, Mexico's Angry Novelist", Books Abroad, XXXVIII, No 4 (Autumn I964), 380-382.

25. Mendoza, María Luisa, "Carlos Fuentes el mexicano", Excélsior, 6 diciembre de I959.

26. Menton, Seymour, "Genesis of a Power Elite", Saturday Review, XLVII (May 23, I964), 44.

27. Millán, María del Carmen, "Sobre Carlos Fuentes, La muerte de Artemio Cruz", Revista Iberoamericana, XXVIII (I962), 397-399.

28. Novo, Salvador, "Ventana, Carlos Fuentes, una revelación", Novedisdes, 6 de junio de 1958.

29. Pacheco, J. E., "Reseña de La región más transparente", Estaciones, III, $\mathrm{N}^{\circ}$ ro (1958), I93-I96.

30. "Rapacious Rebels", reseña anónima, Newsweek, May i8, i964, p. I Io (sobre The Death of Artemio Cruz).

3I. Reyes Nevares, Salvador, "Notas sobre La región mäs trinsparente", Estaciones, III, No to (I958), I65-I77.

32. Rodman, Selden, "The Heroes Must Fail", New York Times Book Review, Nov. 13, 1960, p. 44 (sobre Where the Air Is Clear).

33. - - "Revolution Isn't Enough", New York Times Book Review, Nov. 12, I961, p. 4 (sobre The Good Conscionce).

34. Sánchez Palacos, Juan Angel, "Algunos aspectos de la novela 'La muerte de Artemio Cruz" ", Armas y Letras, VI, No 4 (I963), 83-89.

35. Sommers, Joseph, "Novels of a Dead Revolution", Nation, CXCVII (June I963), Ir4-II5.

36. - "Sobre Carlos Fuentes, 'La muerte de Artemio Cruz" ", His. panis, XLVI (1963), 856-857.

37. Stevenson, Philip, "The Very Navel of the Star", Mainstream, XIV, No 7 (1960), 42-49 (sobre Where the Air is Clear).

38. Torres-Rioseco, Arturo, "Spanish American Novelists of Today", Aspects of Spanish American Literature (University of Washington Press. 1963), pp. 72-90. 
39. Valenzuela, Víctor M., "Carlos Fuentes, novelista mexicano", La Voz, VII, No 2 ( 1962 ), 10-13.

40. Vázquez Amaral, José, "Mexico's Melting Pot", Saturday Revieu', XIIII (Nov. 19, 1960), 29.

4r. Velarde, Agustín, Carlos Fuentes y Las buenas conciencias (México: Buena Prensa, A. C., r962).

42. Villaseñor, R., "Sobre Carlos Fuentes, 'La muerte de Artemio Cruz'", Vida Universitaria, XII, No 594 (1962), p. 3 у p. Io.

43. West, Anthony, "The Death of Artemio Cruz", New Yorker, Aug. 8 , 1964 .

44. "The Whole of Life", reseña anónima, The New Yorker, March 4, 1961, pp. I23-ז26 (sobre Where the Air Is Clear).

Carleton University

RICHARD L. JACKSON 
